\title{
ANIMATED VECTORS FOR VISUALIZATION OF POWER SYSTEM PHENOMENA
}

\author{
J. Gronquist W. Sethares F. Alvarado R. Lasseter \\ Department of Electrical and Computer Engineering \\ The University of Wisconsin
}

\begin{abstract}
This paper presents an exact mechanical ana$\log$ for an electric power system that proves to be a powerful visualization tool for studying power systems issues such as load flow, transient stability, and the effects of FACTS devices. Techniques based on color and highlighting that make the method applicable to the visualization of large systems are introduced. The analog provides a means for demonstrating complex issues to audiences with little or no background in power systems. For the power engineer, the analog provides unique insights into the hows and whys of power system behavior. To the control center operator, an accurate model of the dynamic modes may bring about improved understanding of system behavior after contingencies and illustrate the relative merits of various dispatch options.

Keywords: Mechanical analog, Visualization, FACTS devices, Load flow, Transient Stability.
\end{abstract}

\section{Introduction}

To the uninitiated, power systems often seem to be an intractably complicated morass of equations and ad hoc rules of thumb. Would five minutes be enough to explain to an engineer without a strong background in power systems why capacitors can support bus voltages? Would the explanation be coherent to an educated layman? What pictures or graphs can be used to visualize transient stability phenomena, the effects of varying power demand on bus voltages, or the influence of FACTS devices?

This paper presents a mechanical analog for electric power systems, (similar to that of [2]) which was presented at [4]. The analog proves to be a powerful visualization tool, and this paper develops the analogy further and extends it to considerably larger systems. Recently, the authors discovered that a similar idea us-

This paper was presented at the 1995 IEEE Power Industry Computer Applications Conference held in Salt Lake City, Utah, May 7-12, 1995. ing pivoted arms and springs was introduced in 1926 by $\mathrm{S}$. Griscom [5], who restricted attention to radial systems which could be physically realized with springs and levers. This paper constructs such an analog for an arbitrarily connected network. Since a computer does not have to deal with issues such as how to prevent springs from becoming entangled, it is possible to study much more complicated systems than Griscom could.

In our analogy, generators are represented by masses constrained to move along a family of concentric circles, and transmission lines are represented by linear springs. Electrical current translates into force, and current sources/sinks such as generators and loads correspond to forces pulling upon the network of masses and springs.

Other visualization techniques for power systems dynamics include the "rolling ball" analogy of [3]. Visualization techniques for static system behavior but using animation techniques include techniques for the visualization of voltage sensitivities [6] and visualization of power flows $[7,8,9]$. Animation can be used to effectively describe other aspects of power system behavior.

In this paper, the power system is assumed to have lossless transmission lines, and generators are modeled as constant voltages behind transient reactances. The load model is arbitrary, that is, no restriction on how the load varies as a function of the system state is assumed. We show that the mechanical analog is functionally identical to the power system model.

This analog may prove to be an ideal vehicle for teaching and explaining a wide variety of power system behaviors and problems. In particular, it may be of great value in explaining concepts of power system operation such as transient stability or the effect of FACTS devices to audiences of limited technical expertise. It may also be possible to use this analog in control center applications, to illustrate on line, dynamic effects of anticipated events or actions. When coupled with good graphics and animation, the analog provides unique insight into the hows and whys of issues like transient stability, load flow, and the use of FACTS devices.

\section{The Analog}

Consider a system of masses $M_{i}$, springs $b_{i j}$, and force vectors $\hat{F}_{i}$ and $\hat{T}_{j}$, as in figure 1 . 


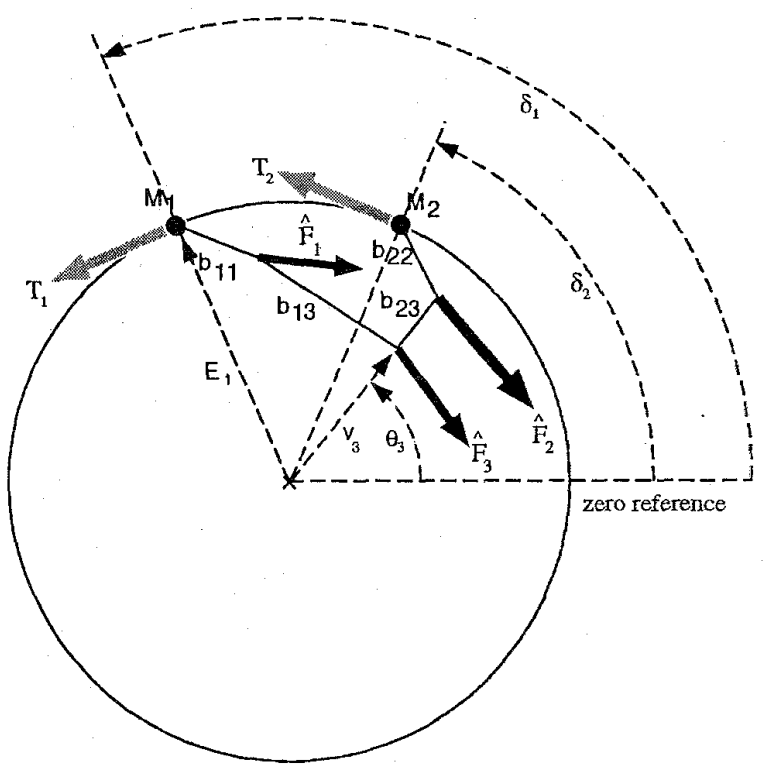

Fig. 1: The mechanical analog for a two generator, three load system.

- The masses slide frictionlessly on concentric circles of radii $E_{1}$ and $E_{2}$. In this case $E_{1}=E_{2}$.

- The mass positions are specified by angles $\delta_{1}$ and $\delta_{2}$ with respect to a zero reference.

- Torques exerted at each node are the angular component of the force vectors acting at each node, times the radial distance. The angular forces acting on the masses, $T_{1}$ and $T_{2}$, are represented by shaded arrows. The torques on masses $M_{1}$ and $M_{2}$ are $\tau_{1}=T_{1} E_{1}$ and $\tau_{2}=T_{2} E_{2}$.

- The masses are connected via a network of springs. A point where springs connect is a 'load node'. The spring between load nodes $i$ and $j$ has force modulus $b_{i j}$.

- The position of load node $k$ is specified by its radial distance from the origin $v_{k}$, and angle $\theta_{k}$.

- The force vectors $\hat{F}_{1}, \hat{F}_{2}$, and $\hat{F}_{3}$ denote the load. The sum of the torques exerted by these forces must equal the sum of the torques applied to the masses for the system to be in equilibrium.

To get a feel for this system, suppose it is initially at rest, with the sum of the torques acting on the system being zero. If one of the forces, say $\hat{F}_{2}$, were to increase, this would have to be countered by an increase in $T_{1}$ and/or $T_{2}$, in order for the system to remain at rest. Increasing these forces stretches the springs and causes the entire system to realign itself in some way. Varying the direction in which $\hat{F}_{3}$ acts, by rotating it clockwise, causes spring $b_{23}$ to stretch and $b_{13}$ to shorten. Tightening a spring (increasing its force modulus) causes a similar realignment. For instance, increasing the force modulus $b_{22}$ will cause $M_{2}$ to shift clockwise. Thus it is fairly easy to gain a qualitative feel for the way various changes will affect the system.

\subsection{The Electrical System Equations}

To see how the analog relates to an electric power system, consider a system of $N_{m}$ generators and $N_{b}$ buses $\left(N_{m} \leq N_{b}\right)$ where :

- the transmission system is lossless

- generators are modeled as a constant voltage behind a transient reactance

- $H_{k}$ is the inertia constant for generator $k$

- $-j b_{k m}$ is the admittance of the $(k, m)$ branch

- $-j b_{k k}$ is the admittance of generator $k$ 's transient reactance

- $P_{k}+j Q_{k}$ is the net load at load bus $k$

- $E_{k}, \delta_{k}$ is the voltage and rotor angle, generator $k$

- $v_{k}, \theta_{k}$ is the voltage and angle for bus $k$.

- $P_{m, k}$ is the mechanical input power to generator $k$

Then the active power injected into bus $k$ from generator $k$ is $P_{k}^{g}=b_{k k} E_{k} v_{k} \sin \left(\delta_{k}-\theta_{k}\right)$, and the reactive power injected into bus $k$ from generator $k$ is $Q_{k}^{g}=b_{k k} v_{k}\left(E_{k} \cos \left(\delta_{k}-\theta_{k}\right)-v_{k}\right)$.

Summing the active power injections into bus $k$ yields

$$
f_{k}=-P_{k}+P_{k}^{g}+\sum_{m=1, m \neq k}^{N_{b}} b_{k m} v_{k} v_{m} \sin \left(\theta_{m}-\theta_{k}\right)=0
$$

Summing the reactive power injections into bus $k$ yields

$g_{k}=-Q_{k}+Q_{k}^{g}+\sum_{m=1, m \neq k}^{N_{b}} b_{k m} v_{k}\left(v_{m} \cos \left(\theta_{m}-\theta_{k}\right)-v_{k}\right)=0$

If we consider the generator terminal voltages to be fixed (the generator terminals are buses $1 \ldots N_{m}$ ), the above equations represent the usual set of load flow equations for a lossless transmission system.

\subsection{The Mechanical System Equations}

In the mechanical system :

- Let $\bar{a}_{v_{k}}$ be the unit vector in the increasing radial direction at load node $k$

- Let $\bar{a}_{\theta_{k}}$ be the unit vector in the increasing angular direction at load node $k$ 

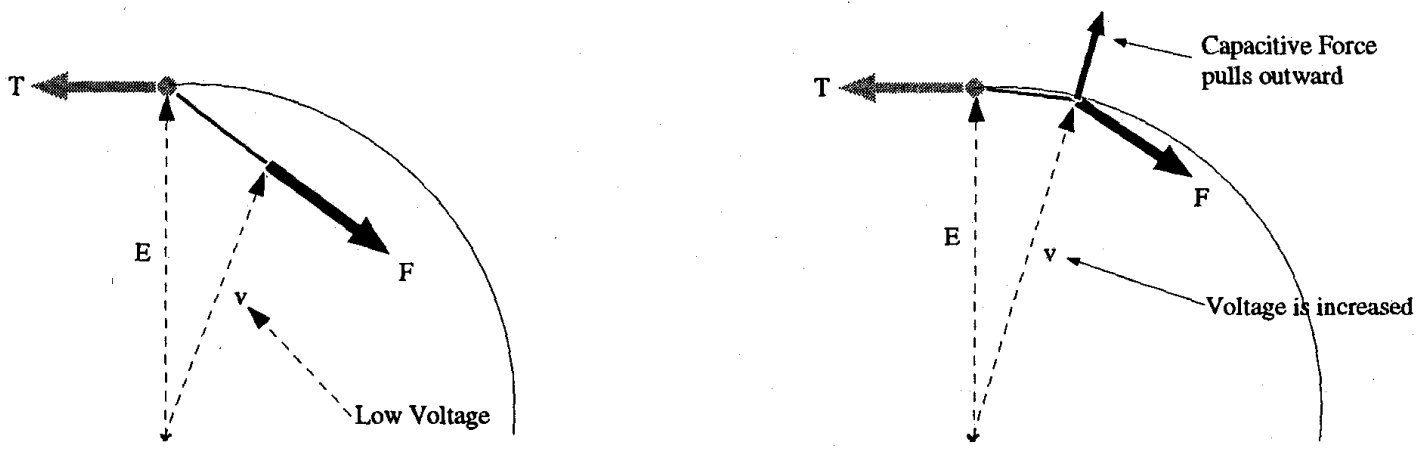

Fig. 2: Illustration of the effect of shunt compensation.

- Let $\bar{a}_{E_{k}}$ be the unit vector in the increasing radial direction at mass $k$

- Let $\vec{a}_{\delta_{k}}$ be the unit vector in the increasing angular direction at mass $k$

Then :

$\bar{a}_{v_{m}} \cdot \tilde{a}_{v_{k}}=\cos \left(\theta_{m}-\theta_{k}\right), \quad \bar{a}_{v_{m}} \cdot \bar{a}_{\theta_{k}}=\sin \left(\theta_{m}-\theta_{k}\right)$

$\bar{a}_{E_{k}} \cdot \tilde{a}_{v_{k}}=\cos \left(\delta_{k}-\theta_{k}\right), \quad \bar{a}_{E_{k}} \cdot \bar{a}_{\theta_{k}}=\sin \left(\delta_{k}-\theta_{k}\right)$

Let each load force $\hat{F}_{k}$ have components $-\frac{P_{k}}{v_{k}}$ in the angular direction and $-\frac{Q_{k}}{v_{k}}$ in the radial direction.

Summing forces at load node $k$ yields :

$$
\begin{aligned}
\Xi_{k}= & -\frac{P_{k}}{v_{k}} \bar{a}_{\theta_{k}}-\frac{Q_{k}}{v_{k}} \bar{a}_{v_{k}}+b_{k k}\left(E_{k} \bar{a}_{E_{k}}-v_{k} \bar{a}_{v_{k}}\right) \\
& +\sum_{m=1, m \neq k}^{N_{b}} b_{k m}\left(v_{m} \bar{a}_{v_{m}}-v_{k} \bar{a}_{v_{k}}\right)=0
\end{aligned}
$$

\subsection{Mechanical versus Electrical Equations}

We claim that the electrical system and the mechanical system are functionally identical. To see this, decompose $\Xi_{k}$ into orthogonal components by :

- Taking the angular component of $\Xi_{k}$ and multiplying by $v_{k}$ yielding $v_{k} \cdot \Xi_{k} \cdot \bar{a}_{\theta_{k}}=f_{k}=0$.

- Taking the radial component of $\Xi_{k}$ and multiplying by $v_{k}$ yielding $v_{k} \cdot \Xi_{k} \cdot \bar{a}_{v_{k}}=g_{k}=0$.

We therefore conclude the equations governing the static equilibria of the two system are identical.

Additionally, consider the power mismatch equation for each generator in the electric power system :

$$
G_{k}=H_{k} \ddot{\delta}_{k}=P_{m, k}-b_{k k} E_{k} v_{k} \sin \left(\delta_{k}-\theta_{k}\right)
$$

Calculating the net angular force exerted on mass $k$ in the analog, and multiplying by $E_{k}$ yields $E_{k}^{2} M_{k} \ddot{\delta}_{k} \equiv$ $H_{k} \ddot{\delta}_{k}=G_{k}$, if we let $\tau_{k}=P_{m, k}$. This implies the two systems are also governed by identical dynamics.

We can use this analog to study both steady state ('load flow') and dynamic ('transient') behavior of electrical power systems.
The 'usual' analog, as in [1], uses a non-linear, nonconservative spring in a 'linear' topology. In this ana$\log$ the non-linearity of the equations is due the 'nonlinearity' of the topology into which the system of linear, conservative springs is embedded. Every quantity in the electric power system can be directly identified with a quantity in the mechanical analog, and many power system devices can be identified with simple mechanical devices. Table 1 shows equivalent quantities.

\begin{tabular}{|c|c|}
\hline Electric Quantity & Mechanical Quantity \\
\hline Active Power $\Leftrightarrow$ & Torque \\
\hline Current Magnitude $\Leftrightarrow$ & Force Magnitude \\
\hline Reactive Current $\Leftrightarrow$ & $\begin{array}{l}\text { Radial Force } \\
\text { (inward is inductive) }\end{array}$ \\
\hline Line Admittance $\Leftrightarrow$ & Spring Force Modulus \\
\hline Voltage Magnitude $\Leftrightarrow$ & Radial Distance from origin \\
\hline Voltage Phase Angle $\Leftrightarrow$ & Node or mass position angle \\
\hline Generator Inertia $\Leftrightarrow$ & Mass moment of inertia \\
\hline
\end{tabular}

Table 1: Equivalence between electrical and mechanical quantities.

FACTS devices can also be represented in terms of the analog. If shunt devices such as SVC (Static Var Compensators) are modeled as strictly reactive elements (such as variable shunt admittances, current sources, or VAR sources) they correspond to forces acting radially at various points in the network. If TCSCs (Thyristor Controlled Series Capacitors) are modeled as variable series admittances, they correspond to springs with varying force moduli.

\section{Examples}

Example 1, The effect of capacitive shunt compensation on load voltage.

Figure 2 shows the effect of capacitive shunt compensation for a one machine, one load system.

The picture on the left shows a situation where load voltage is unacceptably low. Adding capacitive compensation at the load, perhaps with an SVC, corresponds to a force pulling radially outward, elevating 


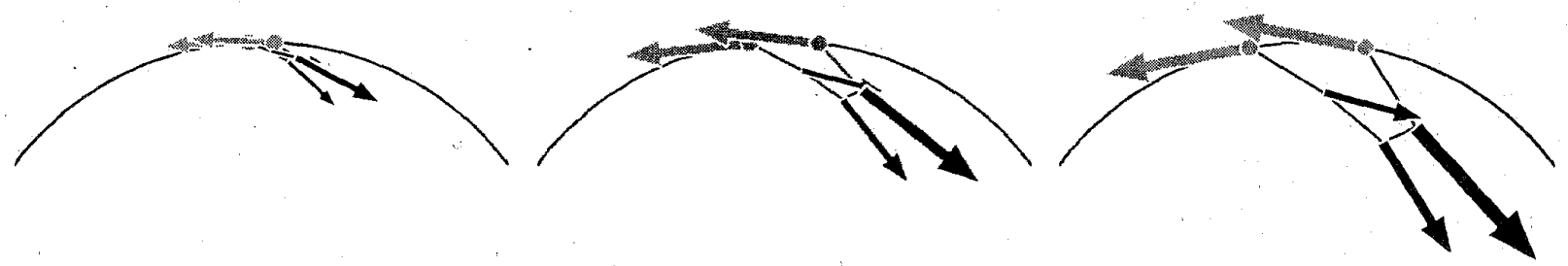

Fig. 3: Effects of increasing load.

load voltage, which can be seen as the radial distance $v$ increasing. Here the total area of the arrow is drawn proportional to load current magnitude, $I$. The length of the arrow is $\alpha \sqrt{\beta I}$ and the width $\frac{1}{\alpha} \sqrt{\beta I}$, where $\alpha, \beta$ are positive constants. The area of the arrow is therefore $\beta I$. Large $\alpha$ gives 'long, skinny' arrows, while small $\alpha$ gives 'short, fat' arrows. $\beta$ scales arrow size uniformly. As voltage increases, the size of arrow $F$ decreases. For this case, $F$ has components $-\frac{P}{v}$ in the angular direction, and $-\frac{Q}{v}$ in the radial direction, with $P$ and $Q$ constant. Therefore, when $v$ increases, the magnitude of $F$ will decrease. This corresponds to the magnitude of the current drawn by a constant $P Q$ load dropping as load terminal voltage increases.

\section{Example 2, Load Flow Visualization}

The effects of increasing load on a system can be seen in figure 3 . The system is the same as that shown in figure 1, two generators supply a system of three loads. Moving from left to right, both the load arrows (black), and the generator arrows (shaded) increase in magnitude, corresponding to a simultaneous increase in total system load and generation. The arrows are drawn as in Example 1. The radial component of the force at the generators is not shown since it is assumed (by the constant voltage behind a transient reactance model) that radial forces have no effect on the generators. This implies the generators have unlimited reactive capability. As load increases, the lines representing the springs get longer. This corresponds to a larger voltage drop across the transmission lines, and implies higher current magnitude in the line. As load increases, the relative angles between points in the network increase with increasing load, confirming the intuition that higher power transfer implies larger angle differences in the network. With increasing load, the radial distance from the origin of the load nodes decreases. This verifies intuition gained from 'nose curves', in that system voltages are expected to begin to drop at some point as load increases.

\section{Example 3, Visualizing Transient Stability}

For this example, consider the system of two generators, one load, and three transmission lines shown in figure 4.

Arrows $T_{1}$ and $T_{2}$ correspond to the mechanical input power to generators $G_{1}$ and $G_{2} . F_{3}$ corresponds to load $P_{3}+j Q_{3}$. In the mechanical analog diagram above, springs $b_{11}$ and $b_{22}$ are much shorter than the other springs in the system as the transient reactances of $G_{1}$ and $G_{2}$ are assumed to be much smaller than the transmission line reactances.

A fault will be applied to the system at the point indicated in figure 4 . This can be visualized as tying this point in the spring directly to the origin, representing zero voltage at the point of the fault.

The effects of the fault can be seen in the sequence depicted in figure 5 .

During the fault, there are net torque (power) imbalances at the masses (generators). This causes one to accelerate, the other to decellerate, with a net effect of an increased angle difference between the masses. During the course of the fault, each mass acquires kinetic energy. If, when the fault clears, the system has enough kinetic energy, the masses will continue traveling past 180 degrees relative angle, as in the last picture. Once the masses pass through 180 degrees, they will accelerate toward each other, increasing system kinetic energy further. Synchronism of the system has been lost.

If the fault were of somewhat shorter duration; the masses could not get to the point of 180 degrees relative angle, as the system would not have enough kinetic energy to stretch the springs this far. For that case, the system would remain synchronized (the relative angle between the masses would remain bounded).

\section{Visualizing Large Networks}

For large networks it is not practical to represent every load, every generator and every line. One option is to use incomplete representations, retaining only a few lines, loads and generators. Unfortunately, the retained network may be disconnected, leading to very poor intuition. Instead, it is possible to use color and/or highlighting to fade into the background less interesting portions of the system, while highlighting either portions of the system of interest (lines, buses), or at least highlighting selected "key" buses.

The technique is surprisingly effective. Figure 6 illustrates the visualization of a large network and the use of highlighting techniques for dealing with large systems. These displays correspond to a 1300 bus system at two different load levels. 


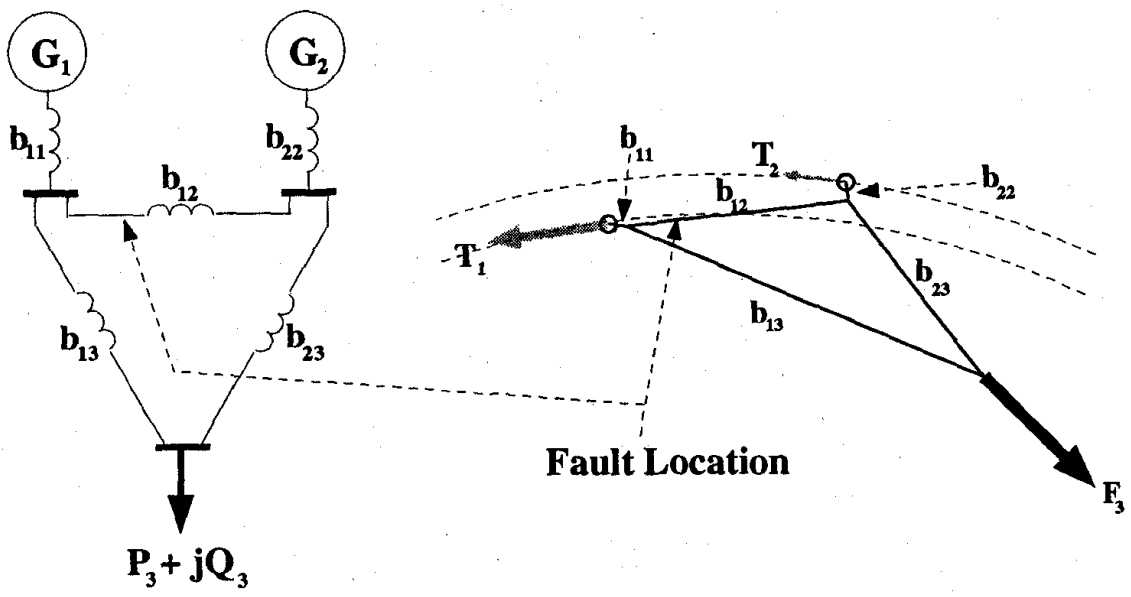

(a) One Line Diagram

(b) Steady State Mechanical Analog

Fig. 4: Pre-fault equilibrium conditions for a two generator, one load system.

\section{Further Extensions}

So far machine damping has not been included in the model presented here. Damping can be included by imagining the entire system of masses and springs being immersed in a viscous fluid, such as oil or water. Machine damping constants can then be thought of as proportional to mass cross-sectional area.

For systems with transmission losses, the analog breaks down as the mass/spring system and the electrical power system are no longer functionally identical. For this case, the analog can still be a useful tool. The mechanical analog display shows the electrical distribution of the system, as opposed to one-line diagrams, which show the spatial distribution. The mechanical analog diagrams show the strength or weakness of electrical couplings in the system.

\section{Conclusions}

This paper has presented a new method for visualization of electric power systems problems. With the current state of computer graphics, the analog presented can be refined to yield a visualization tool which enhances the understanding of power system static and dynamic behavior.

Islanding and coherency is particularly easy to visualize, even on large systems. When animated, these diagrams clearly show groups of machines swinging together. Exactly what tie lines could be compensated to more directly couple the coherent groups are easily identified via use of the analog.

In one compact display, these diagrams show magnitude and phase of all voltages in the system, and magnitude and phase of all load currents. Additionally, voltage drops across all transmission lines are evident.
Clearly, the lack of color and animation within the printed page has rendered our displays far less effective than they are in practice. The authors intend to make the results available for viewing via the Internet (the World Wide Web).

\section{Acknowledgments}

The authors wish to gratefully acknowledge C. Taylor for pointing out that mechanical analogs of this type have been built for small systems and that at least one of them has survived to the present.

The authors also acknowledge financial support from NSF grant ECS-9216520 and EPRI contract RP803002 .

\section{REFERENCES}

[1] A. Bergen, "Power Systems Analysis," Ch. 9, Sec. 3,pp. 264-265, Prentice-Hall, 1986

[2] G. Lüders, "Transient Stability of Multimachine Power Systems via the Direct Method of Lyapunov," IEEE Transactions on Power Apparatus and Systems, Vol. PAS-90, No. 1, Jan/Feb 1971, pp. 23-36

[3] T. Athay, R. Podmore, S. Virmani, "A Practical Method for the Direct Analysis of Transient Stability," IEEE Transactions on Power Apparatus and Systems, Vol. PAS-98, No. 2, Mar/Apr 1979, pp. 573-584

[4] J. Gronquist, W. Sethares, "A New Visualization Tool for Power Systems Education and Research," NAPS Proceedings, Manhattan, Kansas Oct. 1994

[5] S. B. Griscom, "A Mechanical Analog to the Problem of Transmission Stability," The Electric Journal, Vol. XXIII, No. 5, May 1926, pp. 230-235

[6] F. L. Alvarado, Y. Hu, C. Rinzin, R. Adapa, "Visualization of spatially Differentiated Security Margins," Proceedings of 1993 PSCC, Avignon, France 


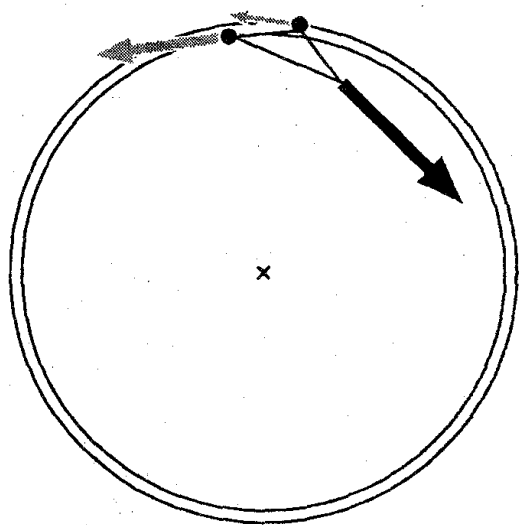

Equilibrium, pre-fault

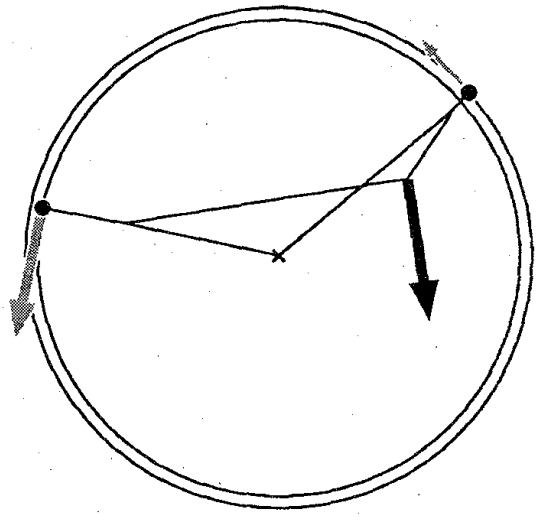

Just prior to fault clearing

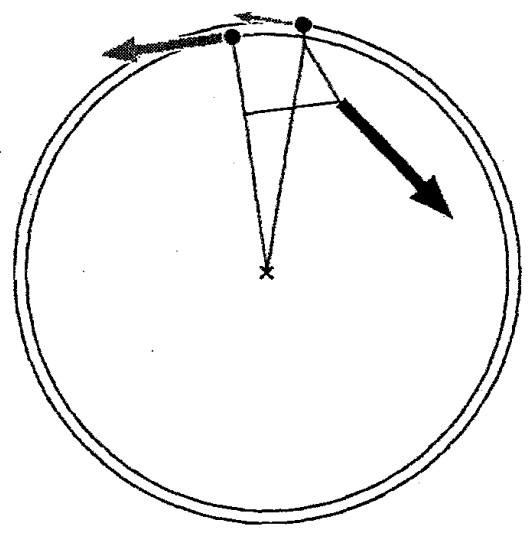

Fault on

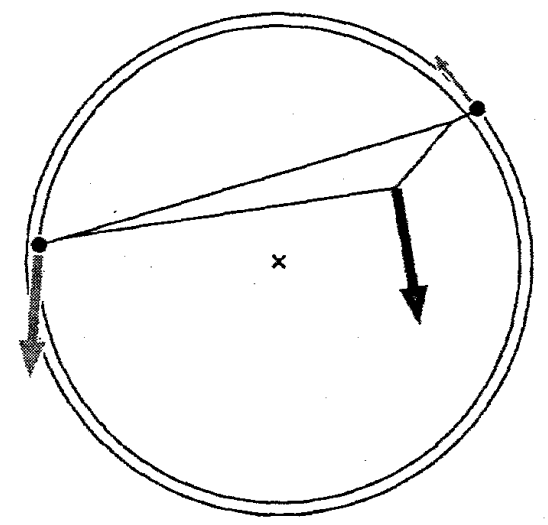

Fault clears

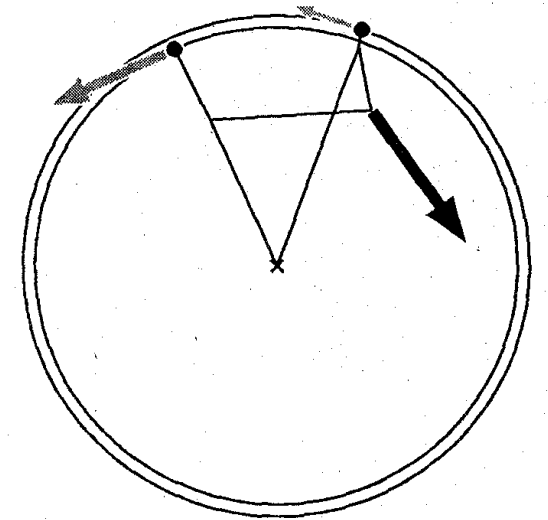

Masses separate

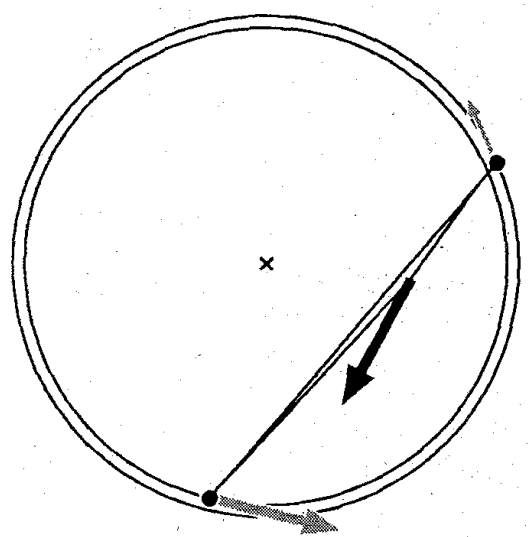

Synchronism is lost

Fig. 5: Mechanical analog visualization of transient behavior.

[7] F. L. Alvarado, "Animation and Visualization of Power Systems," Proceedings of the EPRI Conference on Visualization, Palo Alto, California, Oct. 25-26, 1993

[8] P. Mahadev, R. Christie, "Envisioning Power System Data: Concepts and a Prototype System State Representation," IEEE Transactions on Power Systems, Vol. 8, no. 3, Aug. 1993, pp. 1084-1090

[9] M. Anderson, et. al. "Advanced Graphics for Power System Operation," IEEE Computer Applications in Power, April 1993, p. 25

James F. Gronquist was born in Cloquet, Minnesota in 1963. He received the BS degree in electrical engineering from the University of Colorado in Boulder, and the MS degree in-electrical engineering from the University of Wisconsin in Madison, where he is presently a $\mathrm{PhD}$ candidate. His research interests include analysis of nonlinear systems, power systems control, cellular automata, and application of genetic algorithms for optimization and control.

William A. Sethares received the BA degree in mathematics from Brandeis University, and the MS and PhD degrees in electrical engineering from Cornell University. He has worked at the Raytheon Company as a Sys- tems Engineer and is currently with the Department of Electrical and Computer Engineering at the University of Wisconsin in Madison. His research interests include adaptive systems, and applications of system identification techniques to control, power, and semiconductor processes.

Fernando L. Alvarado was born in Lima, Peru. He received the $\mathrm{BEE}$ and $\mathrm{PE}$ degrees from the National University of Engineering in Lima, Peru, the MS degree from Clarkson University, and the $\mathrm{PhD}$ degree from the University of Michigan in 1972. Since 1975 he has been with the University of Wisconsin in Madison, where he is a Professor of Electrical and Computer Engineering. His main areas of interest are computer applications to power systems and large sparse matrix problems.

Robert H. Lasseter received the $\mathrm{PhD}$ degree in Physics at the University of Pennsylvania, Philadelphia in 1971. He was a Consultant Engineer at General Electric Company until he joined the University of Wisconsin-Madison in 1980. His main interest is the application of power electronics to utility systems. 


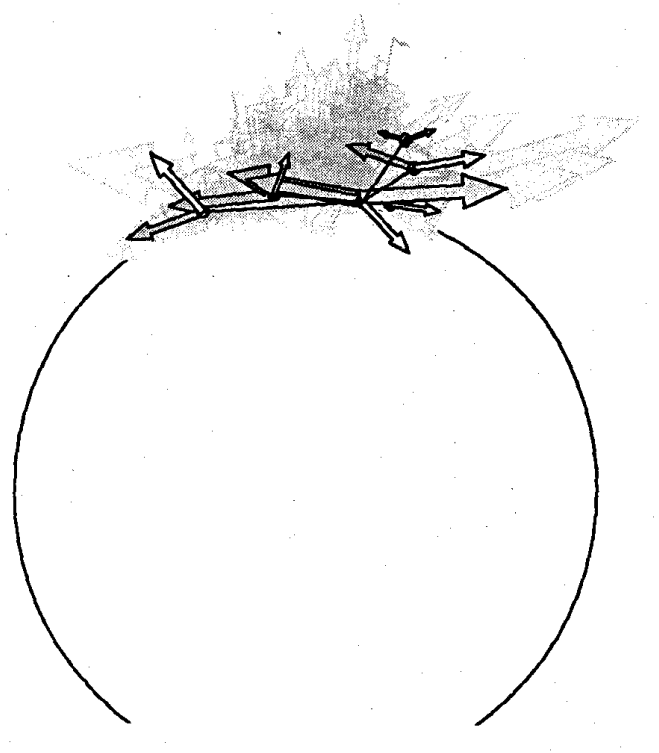

A: Low Load Level

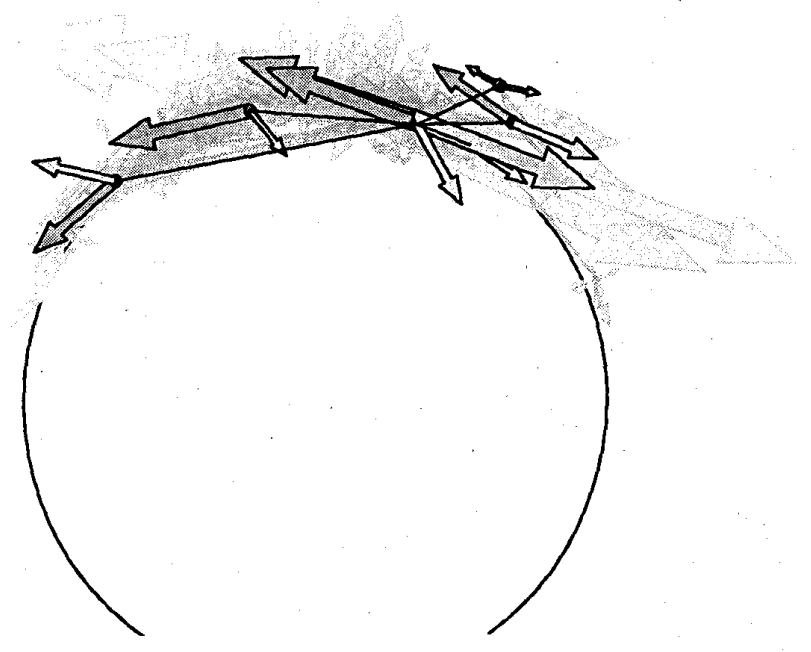

B: High Load Level

Fig. 6: Representation of a 1300 bus system at low and high load levels. 